Audiology

Neurotology
Audiol Neurotol 2006;11(suppl 1):57-62

DOI: $\underline{10.1159 / 000095615}$
Received: March 15, 2006

Accepted: May 10, 2006

Published online: October 6, 2006

\title{
Combined Electroacoustic Stimulation in Conventional Candidates for Cochlear Implantation
}

\author{
Chris J. James $^{a} \quad$ Bernard Fraysse $^{a} \quad$ Olivier Deguine $^{a} \quad$ Thomas Lenarz $^{b}$ \\ Deborah Mawman $^{c}$ Ángel Ramos ${ }^{d}$ Richard Ramsden $^{c}$ Olivier Sterkers ${ }^{e}$ \\ ${ }^{a} \mathrm{CHU}$ Purpan, Toulouse, France, ${ }^{\mathrm{b}}$ Medizinische Hochschule, Hannover, Germany, ${ }^{\mathrm{C}}$ Manchester Royal Infirmary, \\ Manchester, UK, dUniversidad de Las Palmas, Las Palmas, Spain, ${ }^{\mathrm{e} A P-H P ~ B e a u j o n, ~ C l i c h y, ~ F r a n c e ~}$
}

\section{Key Words}

Cochlear implant $\cdot$ Residual hearing $\cdot$ Speech recognition in noise

\begin{abstract}
Objective: To report on combined ipsilateral electrical and acoustic stimulation in a subset of conventional candidates for cochlear implantation where preoperative pure tone thresholds were $\leq 60 \mathrm{~dB} \mathrm{HL}$ for 250 and $500 \mathrm{~Hz}$. Methods: Subjects were 10 adults implanted with the Nucleus 24 Contour Advance perimodiolar electrode array. Soft surgical procedures were strictly observed: $\leq 1.5-\mathrm{mm}$ cochleostomy hole placed anterior and inferior to the round window, a Healon bubble placed over the opening to prevent entry of foreign bodies, and no suction applied. The electrode array was inserted $17 \mathrm{~mm}$ to the first marker rib using the recommended 'advance-off-stylet' technique. Pure tone hearing threshold levels were recorded pre-, and postoperatively at 1-2 and 6-12 months. Speech recognition was tested for cochlear implant $(\mathrm{Cl})$ alone and combined with ipsilateral hearing aid for 7 subjects who retained significant residual hearing in the implanted ear at 1-2 months after operation. Results: There were 3/10 cases where 1-2 months after operation low-frequency responses were considered vibrotac-
\end{abstract}

\section{KARGER}

Fax +41613061234 E-Mail karger@karger.ch www.karger.com
(C) 2006 S. Karger AG, Basel

$1420-3030 / 06 / 0117-0057 \$ 23.50 / 0$

Accessible online at:

www.karger.com/aud tile only (>85-110 dB HL, 250-500 Hz). In the remaining 7 cases, residual hearing was maintained up to at least 6 months after operation with minor changes. Insertion depth angles in these cases ranged from 285 to $420^{\circ}$. For these subjects, the mean preoperative score for words presented at $65 \mathrm{~dB}$ SPL was 22\%. Mean postoperative scores were $56 \%$ for $\mathrm{Cl}$ alone, and $68 \%$ for $\mathrm{Cl}$ plus ipsilateral hearing aid $(\mathrm{p}<$ 0.05 , paired t). For sentences presented in multitalker babble noise at $5 \mathrm{~dB}$ SNR, mean scores were $61 \% \mathrm{Cl}$ alone, and $75 \%$ $\mathrm{Cl}+$ IpsiHA ( $\mathrm{p}<0.01$, paired $t)$. Conclusions: Hearing was conserved during surgery and over time in $70 \%$ of conventional candidates implanted with the Nucleus 24 Contour Advance $\mathrm{Cl}$ who had significant levels of preoperative low-frequency residual hearing ( $\leq 60 \mathrm{~dB} \mathrm{HL}$ ). These conventional candidates for $\mathrm{Cl}$ also benefited from improved speech recognition in noise when using combined ipsilateral electrical and acoustic stimulation.

Copyright $\odot 2006$ S. Karger AG, Basel

\section{Introduction}

Combined electrical and acoustic stimulation via cochlear implant (CI) and hearing aid (HA) has been shown to give added benefits in terms of speech recognition in

E-Mail chris.j.james@wanadoo.fr 
quiet and in noise and for sound quality. Such benefits appear to be accessible for bimodal stimulation using both contralateral residual hearing [Armstrong et al., 1997; Dunn et al., 2005; Ching et al. 2004; Kong et al. 2005] or for 'electroacoustic' stimulation, or bimodal hearing with ipsilateral residual hearing [von Ilberg et al., 1999; Gantz and Turner, 2003; Kiefer et al., 2004; Gstoettner et al., 2004; James et al., 2005; Fraysse et al., 2006; Skarzynski et al., 2003].

A prerequisite for combined ipsilateral electroacoustic stimulation (EAS) is sufficient residual hearing after cochlear implantation. However, at this stage it is not clear what minimum level of residual hearing is required for speech recognition benefits to outweigh the practical disadvantages of additional use of one or more HAs with a CI. It appears that the question cuts two ways; a good level of residual hearing would tend to provide 'unaided' benefits, or, a very poor level of residual hearing could result in physical or loudness discomfort.

Due to studied improvements in the performance of patients with CIs, candidacy criteria have changed over the years from total deafness to a low level of open-set recognition of words or sentences. For example, the limit for candidacy in Germany is 30\% monosyllabic word score, in France and Spain 50\% disyllabic word score, and in the UK 50\% sentence score [Fraysse et al., 2006]. With these criteria CIs are still shown to provide significant improvements in health utility [UK Cochlear Implant Study Group, 2004a] and are cost effective [UK Cochlear Implant Study Group, 2004b]. In these 'conventional' cases where preoperative scores are not negligible, the remaining 'acoustic' function of the implanted ear would otherwise be destroyed by the introduction of the electrode array unless specific precautions are followed.

In the present report, we analyze and present a subset of data collected in a larger study of conservation of residual hearing after cochlear implantation with a perimodiolar electrode, which is most often used with 'conventional' candidates for cochlear implantation. The progress of the multicenter study has been reported by James et al. [2005] and updated more recently by Fraysse et al. [2006]. The latter report identified that specific 'soft' surgical procedures should be adhered to in order to better preserve residual hearing after implantation. The inclusion criteria for this study included a minimum level of preoperative speech perception to be present in the ear to be implanted. There was no limitation placed on pure tone hearing threshold levels. However, in order to effectively aid residual hearing with a high- power in-the-ear (ITE) HA in combination with CI, maximum postoperative thresholds were limited to $80 \mathrm{~dB} \mathrm{HL}$ for 125 and $250 \mathrm{~Hz}$ and $90 \mathrm{~dB} \mathrm{HL}$ at $500 \mathrm{~Hz}$. These limits corresponded to the maximum output characteristics of the Phonak Aero (or Valeo) 33 ITE and to vibrotactile sensations. Where 'soft' surgery was observed, median threshold increases at 1 month after operation were 15, 18 and $25 \mathrm{~dB}$ for 125, 250 and $500 \mathrm{~Hz}$. In addition, there was some further degradation of thresholds in some cases over time and this appeared to happen more often where thresholds were poorer to start with. It was obvious that patients who started with thresholds at the limit of HA performance (e.g. above) would not be candidates for EAS; in addition, in about half of the cases threshold shifts would be greater than $20 \mathrm{~dB}$.

For the current report, cases were selected from the larger study population where preoperative HTLs were $\leq 60 \mathrm{~dB} \mathrm{HL}$ at 250 and $500 \mathrm{~Hz}$. These levels might afford the subject some benefit either with a naked ear or from amplification with ITEs. Also, after discussion of the data with the study group and other experts in the field it appeared a nominal practical criterion where residual hearing may still be considered important in the light of cochlear implantation.

In the present data set, the additional selection criterion was strong adherence to the surgical protocol described by Fraysse et al. [2006] and shown to improve hearing conservation. One surgical criterion was relaxed; that is that the cochleostomy hole size could be up to $1.5 \mathrm{~mm}$ in diameter. Otherwise any other deviation removed the patient from the current subject group. Subjects were implanted at the centers included in the author list.

\section{Methods}

Subjects were 10 adults implanted with the Nucleus 24 Contour Advance perimodiolar electrode array. According to the following criteria, these 10 subjects were selected from the larger study population of 37 patients implanted at the time of writing. Preoperative hearing threshold levels were $\leq 60 \mathrm{~dB}$ HL at 250 and $500 \mathrm{~Hz}$. Specific 'soft' surgical procedures were strictly observed as specified by James et al. [2005] and Fraysse et al. [2006]: A $\leq 1.5-\mathrm{mm}$ cochleostomy hole was made anterior and inferior to the round window, a Healon bubble was placed over the opening to prevent entry of foreign bodies. Suction was avoided at this stage to prevent loss of perilymphatic fluid. The electrode array was inserted $17 \mathrm{~mm}$ to the first marker rib using the recommended 'advance-off-stylet' technique. 
Preoperative hearing threshold levels were roughly symmetrical $(\approx 10 \mathrm{~dB})$ for all subjects. Thresholds were more or less stable over 1-2 months preoperatively. Etiology of deafness was unknown except for P19 who had some family history of hearing loss. All subjects, except P18 due to preference, were implanted in the worse ear according to thresholds $\geq 500 \mathrm{~Hz}$. All subjects were refitted with state-of-the-art digital Phonak Aero/Valeo 33 ITE HAs at least 1 month prior to implantation. Subjects who generally wore only one HA (in the better ear) were given at least 2-month experience with the new bilateral aids. Equivalent performance with old versus new HAs was observed within this period.

Pure tone hearing threshold levels were recorded preoperatively, and postoperatively at 1-2 months and at 6 months or 12 months. Speech recognition was tested with 7 subjects with postoperative residual hearing as according to the criteria $\leq 80 \mathrm{~dB} \mathrm{HL}$ at 125 and $250 \mathrm{~Hz}$.

Postoperatively, subjects were given two types of speech processor program to evaluate; 'overlapping' and 'nonoverlappingshifted'. The former maps the entire frequency range 120 $8000 \mathrm{~Hz}$ across the array 18-22 active electrodes as with conventional CI. The latter used the same filter bands, but one to three low-frequency bands were deactivated so that there was no overlap for frequencies where thresholds were $\leq 80 \mathrm{~dB}$ HL. In addition, the filter band-to-electrode allocation was shifted apically by the number of deactivated channels. Subjects were given either 'overlapping' or 'nonoverlapping-shifted' MAPs to take home for the 1st and 2 nd months postactivation in a balanced design. For the $3 \mathrm{rd}$ month, they could use either program at will. At the end of the 3rd month, they chose their preferred program which was then re-evaluated at 6 months. There were insufficient data at the time of writing to properly analyze the effect of speech processor program on performance. For the CI alone condition, the better score for either program was used.

For these 7 subjects, speech recognition was tested in quiet with words presented in sound-field at $65 \mathrm{~dB}$ SPL and for sentences presented at $70 \mathrm{~dB}$ SPL in multitalker babble with $5 \mathrm{~dB}$ SNR. In most cases, postoperative testing was performed at 6 months after implantation. Listening conditions were CI alone, with both ears plugged or CI+ipsiHA (e.g. non-implant ear plugged, ipsiHA active).

\section{Results}

Preoperative, and 1- to 2-month and 6- to 12-month postoperative audiograms are presented in figure 1 for all 10 subjects. There were 3 cases (P5, P19, P32) where immediately after operation low-frequency HTLs were considered to be at vibrotactile sensation levels $(>85-110 \mathrm{~dB}$ $\mathrm{HL}, 250-500 \mathrm{~Hz}$ ). In the remaining 7 subjects, there were some changes in postoperative hearing levels over time. HTLs measured between 6 and 12 months showed some recovery of pure tone thresholds for the lowest frequencies 125 and $250 \mathrm{~Hz}$ (notably P6), and some degradation at, for example, $500 \mathrm{~Hz}$ (P9, P18). These variations were reflected in median changes for the group; 26, 34 and $35 \mathrm{~dB}$ before operation to 1-2 months after operation, and 15, 26 and $47 \mathrm{~dB}$ before operation to 6-12 months after operation, for 125, 250 and $500 \mathrm{~Hz}$, respectively.

Insertion depth angles obtained from 'Cochlear View' $\mathrm{X}$-ray images [Xu et al., 2000] are indicated for each case in figure 1. A tip fold-over was observed for P6 resulting in a very low insertion angle of $285^{\circ}$. Otherwise, a large range of angles was observed $323-435^{\circ}$ as previously reported in Fraysse et al. [2006]. There did not appear to be any strong systematic effect of insertion angle on changes in audiograms; however, the largest insertion depth angle of $435^{\circ}$ seen in 2 cases (P5, P19) corresponded to the largest losses of residual hearing.

Percent correct scores for words in quiet are presented in figure 2. For the 7 subjects retaining significant residual hearing the mean preoperative score was $22 \%$ indicating limited open-set speech recognition ability. This was in agreement with audiograms where there would be only very limited or, indeed, no access to high-frequency speech information even with well-fitted HAs.

Postoperative word recognition scores with HA alone for the implanted ear were available for 5/7 subjects (fig. 2). It was not an aim of this study to monitor preservation of residual speech recognition; however, these cases showed that both hearing threshold levels and speech recognition can be preserved even with relatively large insertion depth angles of about $400^{\circ}$ (P9, P37).

At 6 months after operation (fig. 2), mean word scores were $56 \%$ for $\mathrm{CI}$ alone, and $68 \%$ for CI plus ipsilateral HA ( $p<0.05$, two-tailed paired t test). Thus, on average there was considerable benefit from cochlear implantation in terms of speech communication ability, and in addition, added benefit from combined use of the ipsilateral HA.

Most subjects had negligible or nil preoperative scores for sentences presented in multitalker babble noise at 5 dB SNR (fig. 3). Postoperatively, mean scores were $61 \%$ for CI alone, and 75\% for CI+IpsiHA ( $<<0.01$, two-tailed paired t test). This indicated the potential for a substantial level of speech communication in a high level of noise and extra benefit from combined ipsilateral stimulation.

Six subjects preferred the 'nonoverlapping-shifted' program; only S37 preferred the overlapping program. The latter was attributed to the use of CI alone away from work in order to 'rest' both ears. It is of note that this subject would need amplification in order to have access to low frequencies via residual hearing; this is not true, for example, for P6, P8 and P9. 


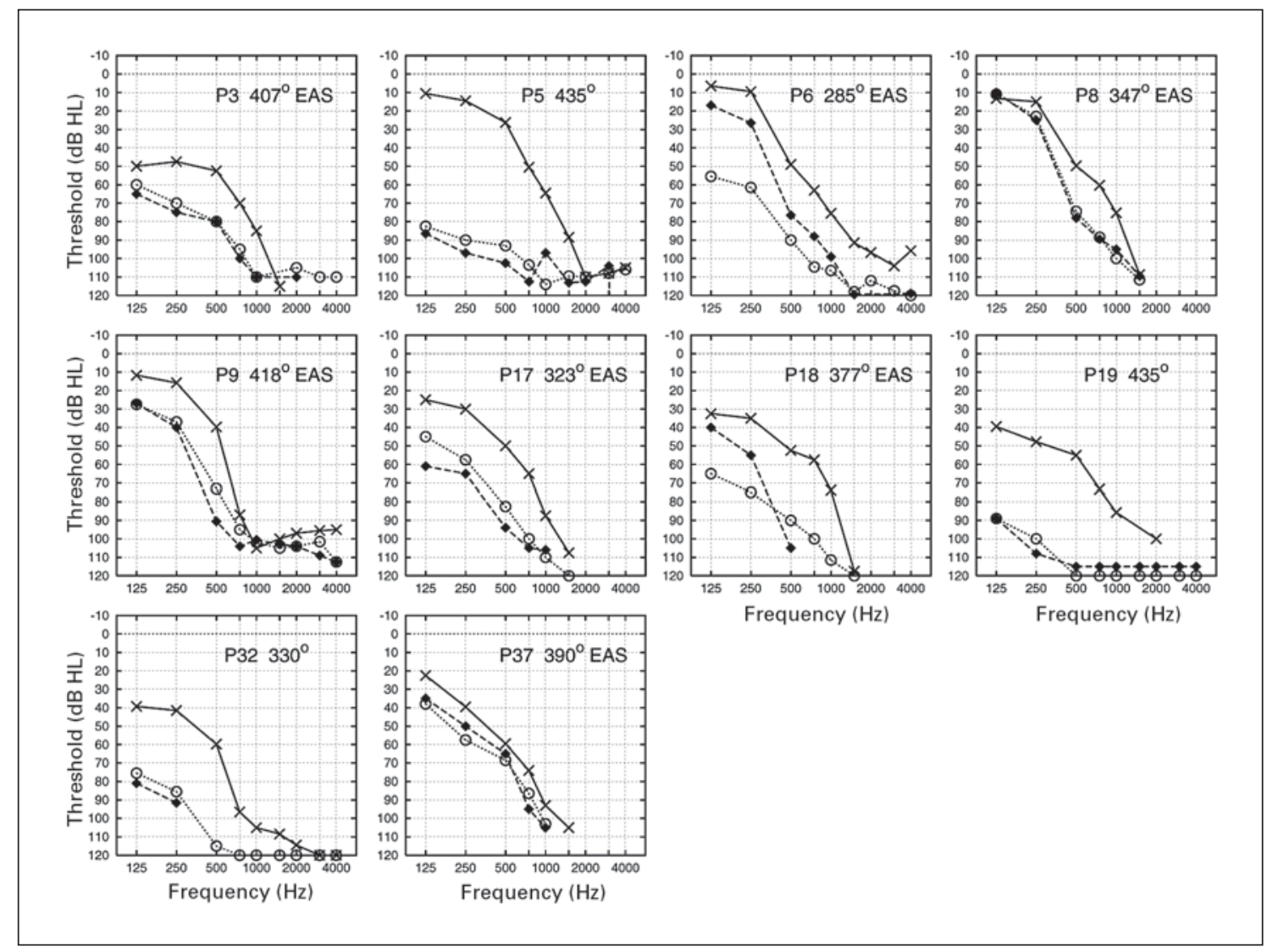

Fig. 1. Pure tone audiograms for 10 subjects implanted with the Nucleus 24 Contour Advance electrode array according to strict 'soft' surgery procedures. Audiograms were measured preoperatively (solid lines, crosses), then at 1 or 2 months after operation (dotted lines, circles) and at 6 to 12 months after operation (dashed lines, filled diamonds). Insertion depth angles measured from X-rays are inset [Xu et al., 2000].

Fig. 2. Individual and mean percent correct recognition scores for lists of words presented in quiet at $65 \mathrm{~dB}$ SPL. Scores are shown for the implant ear only with the contralateral ear plugged. The ipsilateral HA was removed and the ipsilateral ear plugged for the CI alone condition. ${ }^{*} \mathrm{p}<$ 0.05 , significant mean difference, twotailed paired t test. Error bars $=1$ standard deviation. $\mathrm{NA}=$ Not available.

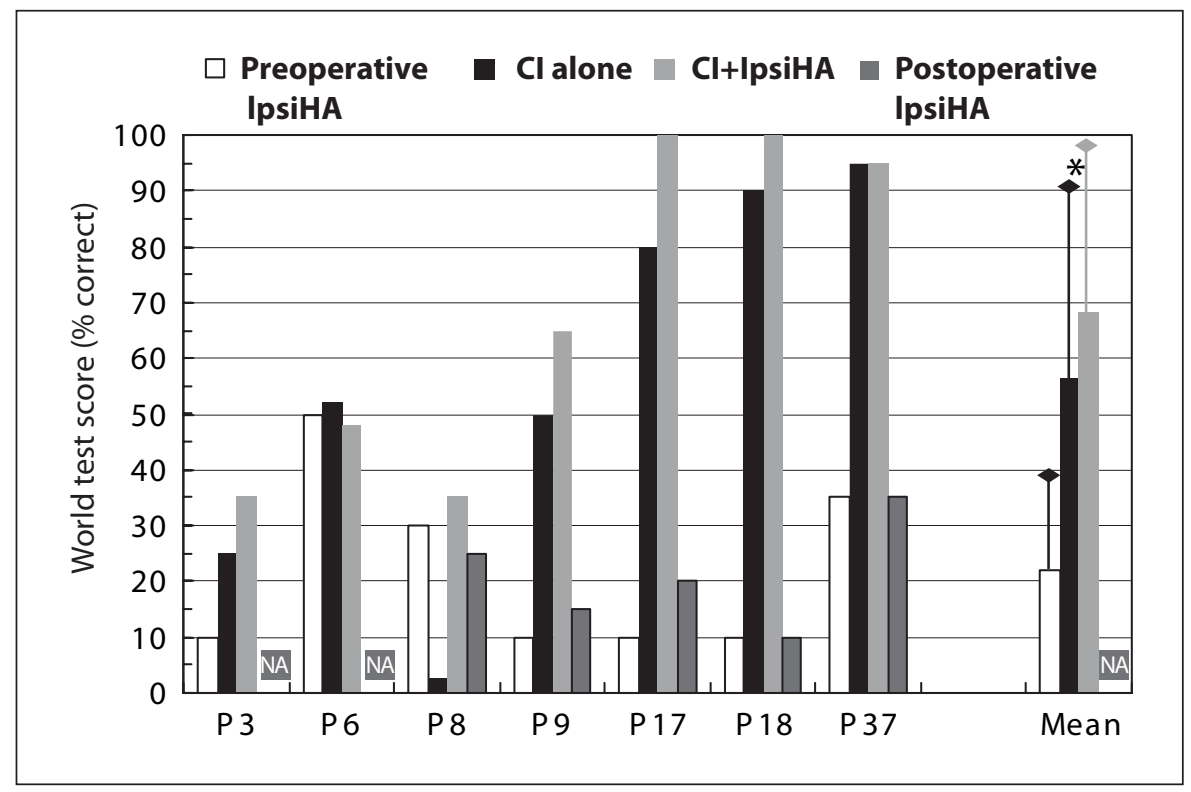


Fig. 3. Individual and mean percent correct word recognition scores for lists of sentences at $70 \mathrm{~dB}$ SPL presented in multitalker babble at $5 \mathrm{~dB}$ SNR. Scores are shown for the implant ear only with the contralateral ear plugged. The ipsilateral HA was removed and the ipsilateral ear plugged for the CI alone condition. ${ }^{* *} \mathrm{p}<$ 0.01 , very significant mean difference, two-tailed paired t test. Error bars $=1$ standard deviation.

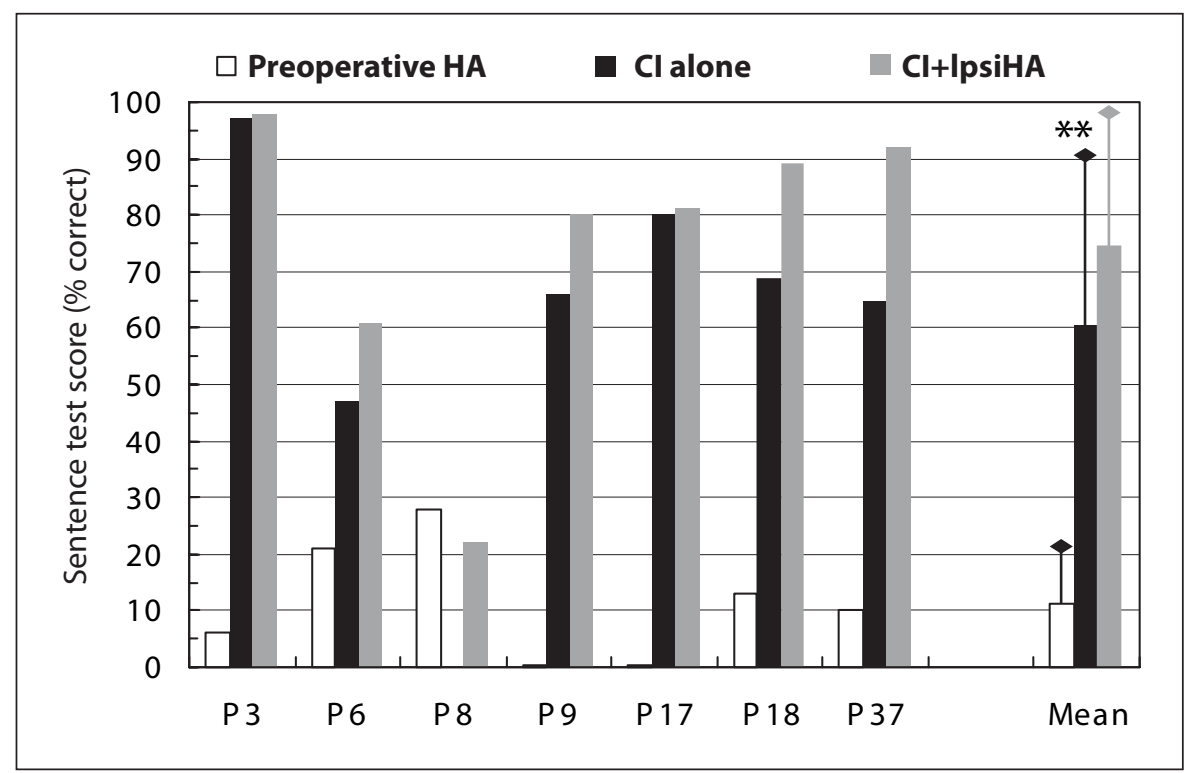

\section{Discussion}

Ten subjects from a large study group of 37 implanted with the Nucleus 24 Contour Advance were selected based on their preoperative HTLs being equal to or better than $60 \mathrm{~dB} \mathrm{HL}$ at 250 and $500 \mathrm{~Hz}$. Useful postoperative residual hearing appeared to be retained in $7 / 10$ or $70 \%$ of cases for at least 6 months. The initial reasons for loss of residual hearing in 3 cases (P5, P19 and P32) remain unclear. Relatively large insertion depth angles of $435^{\circ}$ were seen for P5 and P19, respectively. Insertion depth angles exceeding about $400^{\circ}$ appear to impact residual hearing more negatively when using the Nucleus 24 Contour Advance perimodiolar electrode [Fraysse et al., 2006]. It may be appropriate to further limit insertion depth for small cochleae as suggested by Escudé et al. [this issue, pp. 27-33].

The insertion depth angle for P32 was relatively low $\left(330^{\circ}\right)$; however, the surgeon reported some 'sticking' when moving the electrode off the stylet which may have resulted in increased trauma to the cochlea. It is of note that a tip fold-over did not produce large permanent deterioration in thresholds.

Fraysse et al. [2006] reported some changes in residual hearing over time for a larger data set of 27 cases. Only in 1 case (P12) did they see a substantial deterioration in HTLs over time where initially postoperative levels were sufficient for combined stimulation. This patient had preoperative hearing levels outside the range reported here.

EAS in Conventional CI Candidates
On average, the current group of subjects benefited substantially from cochlear implantation alone in terms of word recognition in quiet. Exceptions were P6 and P8 who had long durations of high-frequency deafness which is known to heavily influence outcomes [Blamey et al., 1996; Yukawa et al., 2004]. However, the combined use of CI with residual hearing allowed P6 a significant level of sentence recognition even in a relatively high level of noise at $5 \mathrm{~dB}$ SNR (fig. 3).

Since the conception of the study, a confounding factor has been identified which appears to influence sentence recognition scores when tested in noise. Where earplugs are used to obtain the CI alone condition, there is the possibility of substantial 'acoustic leak'. This is particularly important where only mild or moderate levels of hearing loss are present in the lowest frequencies in either ear (e.g. P6, P8, P9). Initial results from testing with direct input to the speech processor to obtain a true CI alone condition indicate that the EAS advantage may be much greater than reported here.

Two subjects reported that they did not notice benefit from use of either the ipsilateral (P6) or contralateral (P9) $\mathrm{HA}$ and decided to discontinue use of these after 1 year. This was attributed to negligible gain being prescribed for the lowest frequencies, with only a very narrow band of effective amplification in the slope region of the audiogram.

The preservation of preoperative speech recognition using HA alone indicates that the function of low-frequency hearing may be also be retained after implantation.

Audiol Neurotol 2006;11(suppl 1):57-62 
For the 7 cases who were tested with EAS here, mean 6- to 12-month postoperative HTLs were 36,48 and $84 \mathrm{~dB} \mathrm{HL}$ for 125,250 and $500 \mathrm{~Hz}$ and greater than $95 \mathrm{~dB}$ HL for higher frequencies. Thus, the presence of even rather limited low-frequency acoustic hearing for use with CI seems to provide some access to pitch information which appears to be missing from the electrically coded signal [Kong et al., 2005; Yukawa et al., 2004]. This appears to provide improved speech perception in background noise and improved sound quality either when combined contralaterally [Armstrong et al., 1997; Kong et al., 2005] or here ipsilaterally.

\section{Conclusions}

Hearing was conserved during surgery and over time in $70 \%$ of conventional candidates for cochlear implantation with low-frequency hearing threshold levels better or equal to $60 \mathrm{~dB}$ HL. These conventional candidates for $\mathrm{CI}$ also benefited substantially from improved speech recognition in noise when using combined ipsilateral electrical and acoustic stimulation.

\section{Acknowledgement}

This research is sponsored by Cochlear AG, Basel.

\section{References}

Armstrong M, Pegg P, James C, Blamey P: Speech perception in noise with implant and hearing aid. Am J Otol 1997;18:S140-S141.

Blamey P, Arndt P, Bergeron F, et al: Factors affecting auditory performance of postlinguistically deaf adults using cochlear implants. Audiol Neurootol 1996;1:293-306.

-Ching TY, Incerti P, Hill M: Binaural benefits for adults who use hearing aids and cochlear implants in opposite ears. Ear Hear 2004;25: 9-21.

Dunn CC, Tyler RS, Witt SA: Benefit of wearing a hearing aid on the unimplanted ear in adult users of a cochlear implant. J Speech Lang Hear Res 2005;48:668-680.

Fraysse B, Ramos A, Sterkers O, et al: Residual hearing conservation and electro-acoustic stimulation with the Nucleus 24 Contour Advance cochlear implant. Otol Neurotol 2006;27:624-633.

-Gantz BJ, Turner CW: Combining acoustic and electrical hearing. Laryngoscope $2003 ; 113$ : 1726-1730.

-Gstoettner W, Kiefer J, Baumgartner WD, Pok S, Peters S, Adunka O: Hearing preservation in cochlear implantation for electric acoustic stimulation. Acta Otolaryngol 2004; 124 : 348-352.
-James C, Albegger K, Battmer R, Burdo S, Deggouj N, Deguine O, Dillier N, Gersdorff M, Laszig R, Lenarz T, Manrique Rodriguez M, Mondain M, Offeciers E, Ramos Macias A, Ramsden R, Sterkers O, von Wallenberg E, Weber B, Fraysse B: Preservation of residual hearing with cochlear implantation: How and why. Acta Otolaryngol 2005; 125 : 481-491.

Kiefer J, Gstoettner W, Baumgartner W, Pok SM, Tillein J, Ye Q, von Ilberg C: Conservation of low-frequency hearing in cochlear implantation. Acta Otolaryngol 2004;124:272-280.

Kong YY, Stickney GS, Zeng FG: Speech and melody recognition in binaurally combined acoustic and electric hearing. J Acoust Soc Am 2005;117:1351-1361.

Skarzynski H, Lorens A, Piotrowska A: A new method of partial deafness treatment. Med Sci Monit 2003;9:CS20-CS24.
UK Cochlear Implant Study Group: Criteria of candidacy for unilateral cochlear implantation in postlingually deafened adults. I. Theory and measures of effectiveness. Ear Hear 2004a;25:310-335.

-UK Cochlear Implant Study Group: Criteria of candidacy for unilateral cochlear implantation in postlingually deafened adults. II. Cost-effectiveness analysis. Ear Hear 2004b;25:336-360.

-Vandali AE, Sucher C, Tsang DJ, McKay CM, Chew JW, McDermott HJ: Pitch ranking ability of cochlear implant recipients: a comparison of sound-processing strategies. J Acoust Soc Am 2005;117:3126-3138.

von Ilberg C, Kiefer J, Tillein J, et al: Electricacoustic stimulation of the auditory system. ORL 1999;61:334-340.

-Xu J, Xu SA, Cohen LT, Clark GM: Cochlear view: postoperative radiography for cochlear implantation. Am J Otol 2000;21:49-56.

-Yukawa K, Cohen L, Blamey P, Pyman B, Tungvachirakul V, O'Leary S: Effects of insertion depth of cochlear implant electrodes upon speech perception. Audiol Neurootol 2004; 9:163-172. 